\title{
Influence of oil combinations on the structural properties of oleogels
}

\author{
Yuliya Frolova*, Roman Sobolev, and Alla Kochetkova \\ Federal Research Centre of Nutrition and Biotechnology, Moscow, Russia
}

\begin{abstract}
Studies of the effect of combinations of vegetable oils on the properties of oleogels will provide useful information from a technological viewpoint for the optimal choice of the composition of the oleogel used, as well as regulate the fatty acid composition of food products based on oleogels. This work aimed to study the effect of combinations of sunflower and linseed oils on the properties of oleogels structured with beeswax. As a result of the studies carried out, data were obtained on the change in the structure of oleogels depending on the ratio of oils, which, from a practical viewpoint, in the future, will make it possible to expand the range of food products containing oleogels.
\end{abstract}

\section{Introduction}

Numerous studies of oleogels based on various edible oils (sunflower, olive, rapeseed, coconut, linseed, soybean, rice bran oil, fish oil) with different types of structure-forming agents (wax, monoglycerides, ethylcellulose, etc.) have been performed. Edible oils have different fatty acid compositions [1], which determines their use in oleogels to replace solid fat in the product to reduce the content of saturated and trans-isomeric fatty acids [2] and modification of the fatty acid profile. To improve the fatty acid profile of ready to feed oleogel-based products, linseed oil rich in polyunsaturated fatty acids is used [3]. However, depending on the type of food products, the use of oleogels containing linseed oil in large quantities can lead to changes in sensory profile, which excludes a complete replacement of solid fat in the composition of the products. The results of the analysis of themed scientific publications evidence numerous works on the research of oleogels based on one type of oil, while there are no publications devoted to the research of the properties of oleogels based on combinations of oils. Taking into account the features of each food product, different rheological characteristics of the fat introduced and its substitutes are necessary to achieve the required quality characteristics of the finished products. Therefore, this work aimed to study the influence of combinations of sunflower and linseed oils on the properties of oleogels containing beeswax.

\footnotetext{
* Corresponding author: Y.operarius@yandex.ru
} 


\section{Material and methods}

\subsection{Materials}

In the study, we used: refined sunflower oil (SO) (Russia); linseed oil (LO) (Russia); beeswax (BW) (Russia).

\subsection{Oleogel preparation}

For research, oleogels were obtained by the method [4] based on pure sunflower and linseed oils, as well as based on their combinations (table 1). The amount of structureforming agent, beeswax, was $6 \mathrm{wt} \%$.

Table 1. Formulation compositions of oleogels

\begin{tabular}{|c|c|c|c|}
\hline \multirow{2}{*}{ Type of oleogel } & \multicolumn{3}{|c|}{ Formula composition, \% } \\
\cline { 2 - 4 } & LO & SO & BW \\
\hline OG_1 & 94.0 & - & 6.0 \\
\hline OG_2 & 70.5 & 23.5 & 6.0 \\
\hline OG_3 & 47.0 & 47.0 & 6.0 \\
\hline OG_4 & 23.5 & 70.5 & 6.0 \\
\hline OG_5 & - & 94.0 & 6.0 \\
\hline
\end{tabular}

The prepared oleogels were divided into aliquots for each assay and cooled at a rate of $1{ }^{\circ} \mathrm{C} / \mathrm{min}$, followed by incubation at $23^{\circ} \mathrm{C}$ in a KK 240 climatic chamber (Pol-EkoAparatura, Poland) for 24 hours before the tests.

\subsection{Methods}

\subsubsection{Polarized microscopy}

The morphology of crystals in oleogels was studied using a Zeiss Axio Imager Z1 microscope (Germany). Images were taken with a Plan-Apochromat objective at 20x magnification. Samples of oleogels ( $2 \mu \mathrm{l}$ aliquot) at a temperature of $90^{\circ} \mathrm{C}$ were applied to heated glass slides and covered with a heated coverslip. Cooled at a rate of $1^{\circ} \mathrm{C} / \mathrm{min}$.

\subsubsection{Thermal analysis}

The phase transitions enthalpy $(\Delta \mathrm{H})$, melting points $(\mathrm{Tm})$, and crystallization $(\mathrm{Tc})$ was determined using a DSC 3 differential scanning calorimeter (Mettler-Toledo, Switzerland) equipped with a TC-45-MT intercooler (Huber, Germany) for cooling. Before the study, the rate of heat flow was calibrated using indium. Samples of oleogels 6-8 mg were weighed in $40 \mu \mathrm{l}$ aluminum crucibles (Mettler-Toledo) and sealed. The measuring cycle consisted of five stages: cooling, stabilization, heating, stabilization, and cooling. Heating and cooling were carried out in the range from 10 to $90{ }^{\circ} \mathrm{C}$ at a rate of $10^{\circ} \mathrm{C} / \mathrm{min}$. The data obtained were analyzed using STARe software (Mettler-Toledo). The results are the average values of the thermodynamic parameters measured in duplicate. 


\subsubsection{Texture analysis}

Changes in the texture properties (Hardness (N), Young's Modulus (E') (N / mm2), and Energy $(\mathrm{mJ})$ ) of oleogels were evaluated using a cylindrical probe $10 \mathrm{~mm}$ in diameter on a Shimadzu EZ Test SX universal testing machine (Shimadzu, Japan). The oleogels were prepared by paragraph 2.2 and then poured into $3 \mathrm{ml}$ cylindrical tubes with a volume of 5 $\mathrm{ml}$ and an internal diameter of $14 \mathrm{~mm}$ with a screw cap. The test for the penetration of the cylindrical probe into the sample was carried out at a speed of $5 \mathrm{~mm} / \mathrm{min}$ to a depth of 8 $\mathrm{mm}$. The data were obtained and processed using the Trapezium $\mathrm{X}$ software (Shimadzu, Japan).

\subsubsection{Oil binding capacity (OBC)}

The oil binding capacity of oleogels was determined by centrifugation as described in [5], using a CM-50 centrifuge SIA "ELMI" (Latvia). In a centrifuge tube, previously weighed, were placed approximately $1 \mathrm{~g}$ of oleogel and weighed again. The tubes were centrifuged at $11000 \mathrm{~g}$ for $10 \mathrm{~min}$, and then the supernatant was removed and weighed again. The oil loss (\% released oil) after centrifugation was calculated as the percentage of oil decanted.

\subsubsection{Statistical analysis}

Data processing was carried out using the SPSS Statistics 20 software.

\section{Results and discussion}

According to the chosen technology and formulation (Table 1), experimental samples of oleogels stable at room temperature were obtained (Figure 1).

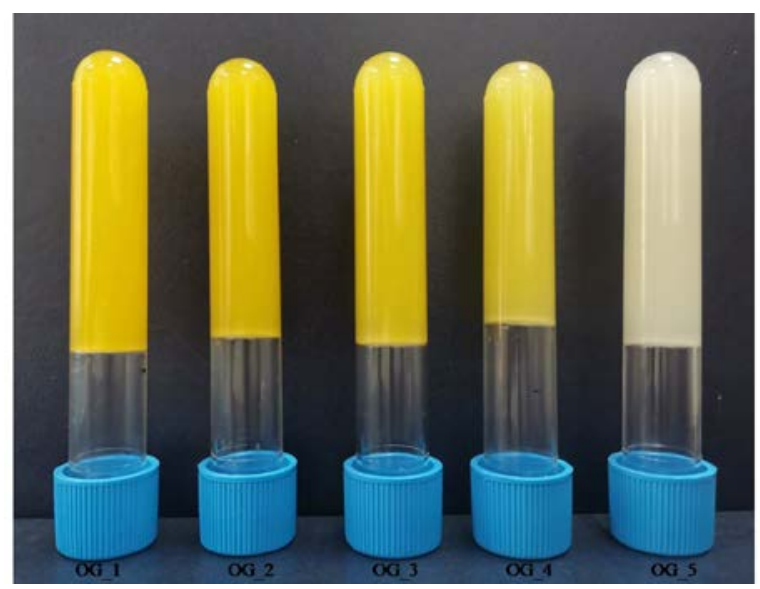

Fig. 1. Appearance of the obtained oleogels

On visual assessment, a change in the color intensity of the oleogels was observed as the content of linseed oil in the composition increased. The use of linseed oil in food technology leads to a change in the color of the ready to feed product (giving a yellow shade) [6], which has an unsatisfactory effect on consumer evaluation. The use of oil combinations in the composition of oleogels makes it possible to reduce the intensity of the yellow color in the ready to feed product, and at the same time ensure high structural properties (data are given below). Studies of the morphology of crystals of oleogels (Figure 2) showed that beeswax crystals in pure linseed oil (Figure 2a) form in the form of 
conglomerates, which decrease as the content of sunflower oil in the oleogels increases (Figure 2c and 2d).

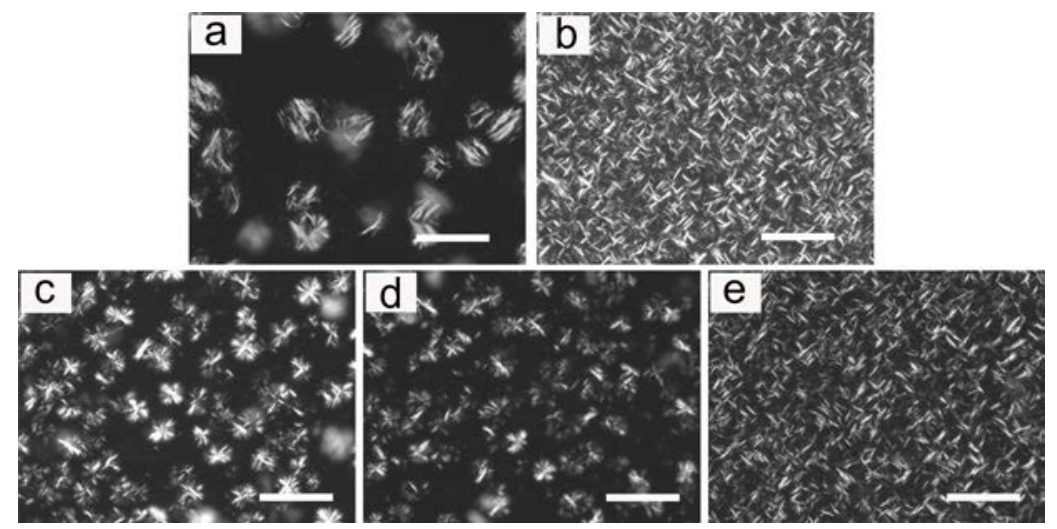

Fig. 2. Microscopy: a - oleogel OG_1;b - oleogel OG_5;c - oleogel OG_2;d - oleogel OG_3;e oleogel OG_4. Magnification 20×.

At an oil ratio of 25:75 (LO: SO), needle-shaped crystals are formed in oleogels, evenly distributed throughout the entire volume of the system (Figure 2e), similar to BW crystals in pure SO-based oleogels (Figure 2b). The morphology, size, and regularity of crystal structure formed in oleogels affect the structural characteristics of finished oleogels [7] and, subsequently, food products containing them.

The thermal properties of oleogels based on pure oils and their combinations, structured with beeswax, were studied using differential scanning calorimetry (DSC) (Figure 3).
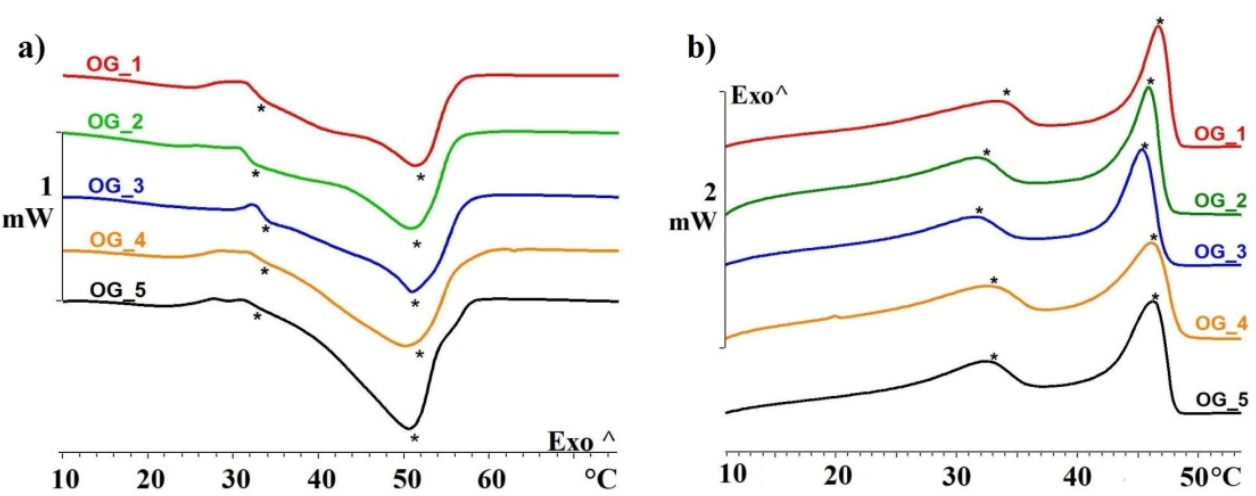

Fig. 3. Curve melting (a) and crystallization (b) DSC of oleogels

As a result of the study, two melting and crystallization peaks were identified in the oleogel. The thermal properties of beeswax, selected as a structure-forming agent, corresponded to the results obtained in previous studies [8]. The presence of several peaks in the oleogel sample may be due to its chemical composition, which contains several components at once with different melting and crystallization temperatures [9]. According to the DSC curves (Figure 3), the melting and crystallization peaks changed slightly depending on the combination of the component composition of the oils used in the oleogel. The thermal effects of wax and oleogels were previously presented in [10, 11], however, data on oleogels based on combinations of these oils are presented for the first time. According to the literature [10], the melting peak of beeswax in the range $40-60{ }^{\circ} \mathrm{C}$ 
corresponds to heat absorption by hydrocarbons and free fatty acids. The second peak is formed due to the absorption of heat by fatty acid esters, which are present in beeswax in large quantities [4]. It is important to note that the data obtained on the thermal properties of the oleogels are largely consistent with the results obtained in the study [11], describing the fact that all the endothermic melting peaks of the oleogels out to be wider than those of the initial structure-forming agent, but had lower values. Table 2 presents the results of determining the phase transitions enthalpy, melting, and crystallization temperatures of oleogels.

Table 2. Thermal properties of oleogels

\begin{tabular}{|c|c|c|c|c|c|c|}
\hline $\begin{array}{c}\text { Type } \\
\text { of } \\
\text { oleogel }\end{array}$ & $\mathbf{T}_{\mathbf{m} 1}$ & $\mathbf{T}_{\mathbf{m} 2}$ & $\Delta \mathbf{H}_{\mathbf{m}}$ & $\mathbf{T}_{\mathbf{c 1}}$ & $\mathbf{T}_{\mathbf{c}}$ & $\Delta_{\mathbf{c}}$ \\
\hline OG_1 & $\begin{array}{c}33,33 \pm \\
0,55\end{array}$ & $\begin{array}{c}51,48 \pm \\
0,90\end{array}$ & $7,33 \pm 0,64$ & $\begin{array}{c}46,70 \pm \\
0,66\end{array}$ & $\begin{array}{c}33,34 \pm \\
0,59\end{array}$ & $7,54 \pm 0,69$ \\
\hline OG_2 & $\begin{array}{c}32,33 \pm \\
0,41\end{array}$ & $\begin{array}{c}51,18 \pm \\
0,22\end{array}$ & $7,77 \pm 0,84$ & $\begin{array}{c}45,86 \pm \\
0,57\end{array}$ & $\begin{array}{c}31,68 \pm \\
0,44\end{array}$ & $9,87 \pm 0,27$ \\
\hline OG_3 & $\begin{array}{c}34,00 \pm \\
0,50\end{array}$ & $\begin{array}{c}50,82 \pm \\
0,75\end{array}$ & $7,32 \pm 0,71$ & $\begin{array}{c}45,36 \pm \\
0,88\end{array}$ & $\begin{array}{c}31,51 \pm \\
0,63\end{array}$ & $8,14 \pm 0,50$ \\
\hline OG_4 & $\begin{array}{c}34,00 \pm \\
0,23\end{array}$ & $\begin{array}{c}51,48 \pm \\
0,14\end{array}$ & $7,17 \pm 0,29$ & $\begin{array}{c}46,02 \pm \\
0,75\end{array}$ & $\begin{array}{c}32,51 \pm \\
0,22\end{array}$ & $8,40 \pm 0,38$ \\
\hline OG_5 & $\begin{array}{c}32,83 \pm \\
0,42\end{array}$ & $\begin{array}{c}50,78 \pm \\
0,56\end{array}$ & $7,30 \pm 0,87$ & $\begin{array}{c}46,19 \pm \\
0,82\end{array}$ & $\begin{array}{c}32,51 \pm \\
0,56\end{array}$ & $7,63 \pm 0,35$ \\
\hline
\end{tabular}

$\mathrm{T}_{\mathrm{m} 1}$ and $\mathrm{T}_{\mathrm{m} 2}$ - melting temperatures of 1 and 2 peaks, respectively; $\mathrm{T}_{\mathrm{c} 1}$ and $\mathrm{T}_{\mathrm{c} 2}-$ crystallization temperatures of 1 and 2 peaks.

The data obtained indicate that the temperature properties of all the samples under study did not differ significantly.

The textural properties, as well as the thermal properties of oleogels, are important factors since they predetermine the quality of the formed systems intended to replace solid fat. Changes in properties characterizing the texture of oleogels depending on the component composition are presented in Table 3. and oil binding capacity is shown in Figure 4.

Table 3. Textural properties of oleogels

\begin{tabular}{|l|c|c|c|}
\hline Type of oleogel & Hardness (N) & $\mathbf{E}^{\mathbf{\prime}}\left(\mathbf{N} / \mathbf{m m}^{\mathbf{2}}\right)$ & Energy $(\mathbf{m J})$ \\
\hline OG_1 & $0,57 \pm 0,03^{\mathrm{a}}$ & $13,15 \pm 7,34^{\mathrm{a}}$ & $2,72 \pm 0,29^{\mathrm{a}}$ \\
\hline OG_2 & $0,52 \pm 0,11^{\mathrm{a}}$ & $10,54 \pm 3,61^{\mathrm{a}}$ & $2,60 \pm 1,04^{\mathrm{a}}$ \\
\hline OG_3 & $0,50 \pm 0,08^{\mathrm{a}}$ & $13,22 \pm 5,51^{\mathrm{a}}$ & $2,53 \pm 0,33^{\mathrm{a}}$ \\
\hline OG_4 & $0,95 \pm 0,16$ & $24,40 \pm 5,56$ & $5,60 \pm 0,35$ \\
\hline OG_5 & $1,62 \pm 0,21$ & $63,73 \pm 27,11$ & $9,14 \pm 1,06$ \\
\hline
\end{tabular}

The letter "a" shows the groups with significant differences compared to the sample OG_5 ( $<<0.01)$

Based on the data obtained, it was found that the samples OG_4 and OG_5 have significant differences in Hardness, Young's Modulus (E'), and Energy compared to other oleogels. 


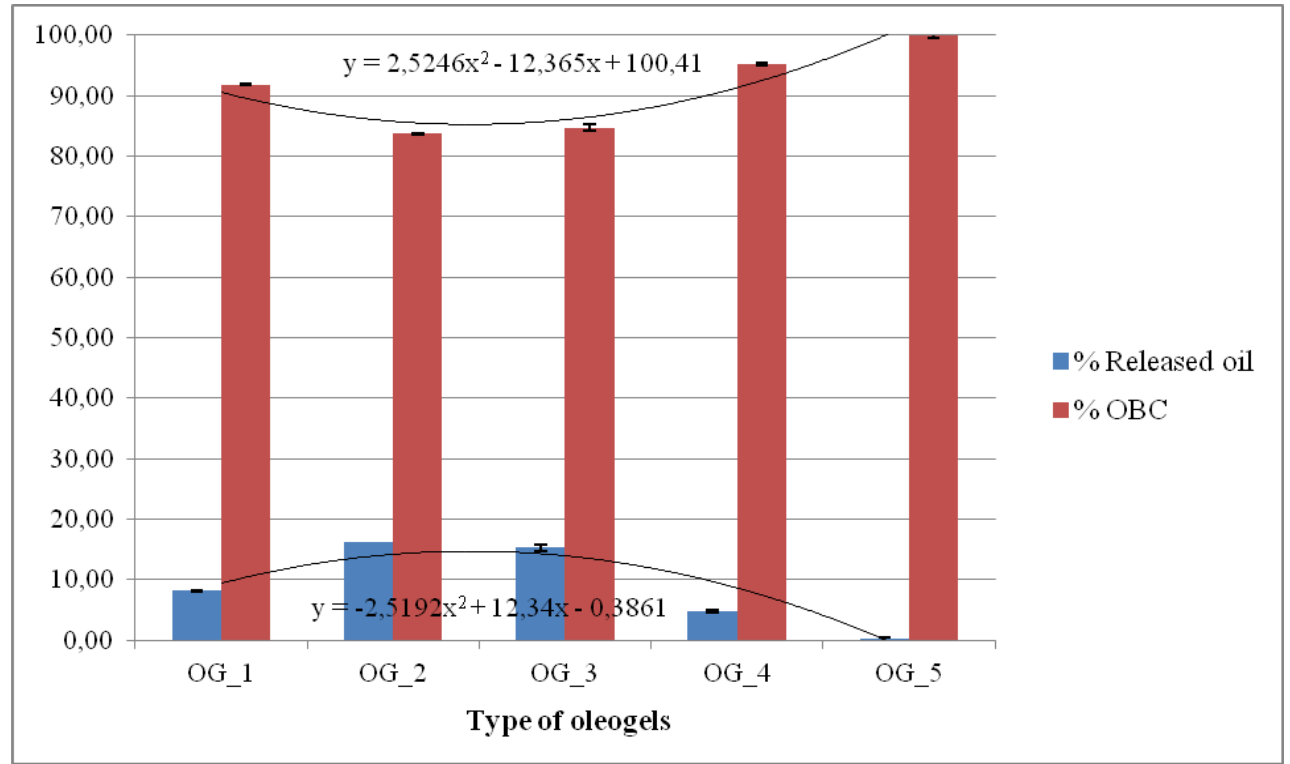

Fig. 4. Oil binding capacity (\% OBC) and \% Released oil from oleogels

It was found that all investigated oleogels have oil binding capacity at a level above 80 \%. In this case, oleogels OG_5 at the concentration of structure-forming agent $6 \%$ under the selected experimental conditions show $100 \%$ oil binding into the oleogel structure. The addition of LO leads to a decrease in the OBC; at the same time, the combination OG_4 has an OBC reliably higher than the OBC for OG_1.

Thus, as a result of the studies carried out, data on the properties of oleogels prepared based on combinations of edible oils structured with beeswax have been obtained for the first time. It was found that the change in the structural properties of oleogels is not linear, depending on the decrease/increase in one of the types of oil in the composition of oleogels. At the same time, the ratio of oils 25:75 (LO: SO) makes it possible to obtain oleogels with better structural characteristics than linseed oleogels, but inferior in properties to oleogels based on sunflower oil. However, the data obtained can be useful from a technological viewpoint when choosing an alternative to solid fats in food products, where oleogels based on pure oils have shown unsatisfactory properties.

The research was supported by a grant from the Russian Science Foundation (Project \#1916-00113).

\section{References}

1. T. Jain, Emerging Technologies in Food Science. (Springer, Singapore, 2020)

2. A. Pușcaș, V. Mureşan, C. Socaciu, S. Muste, J. Foods. 9(1), 70 (2020)

3. D. Franco, A. J. Martins, M, López-Pedrouso, L. Purriños, M. A. Cerqueira, A. A. Vicente, L. M. Pastrana, C. Zapata, J. M. Lorenzo, J. Foods. 8(9), 366 (2019)

4. V. Sarkisyan, R. Sobolev, Yu. Frolova, A. Malinkin, M. Makarenko, A. Kochetkova, J. Am. Oil Chem. Soc. 3, 281-296 (2021)

5. M. Kupiec, A. Zbikowska, K. Marciniak-Lukasiak, M. Kowalska, J. Agriculture, 10(6), 211 (2020)

6. Yu. Frolova, R. Sobolev, V. Sarkisyan, Meat technology, 8, 44-47 (2020) 
7. A. I. Blake, E.D. Co, A. G.Marangoni, J. Am. Oil Chem. Soc. 91(6), 885-903 (2014)

8. Y. Gaillard, A. Mija, A. Burr, E. Darque-Ceretti, E. Felder, N. Sbirrazzuoli, J. Thermochimica Acta. 521(1-2), 90-97 (2011)

9. N. G. Barroso, P. K. Okuro, A. P. B. Ribeiro, R. L. Cunh, Gels. 6, 5 (2020)

10. Z. Ruguo, Z. Hua, Z. Hong, F. Ying, L. Kun, Z. Wenwen, J. Procedia Engineering, 18, 101-106 (2011)

11. G. Fayaz, S. Calligaris, M. C. Nicoli, J. Food Biophysics, 15(1), $42-49$ (2020) 